\title{
Difference image analysis: The interplay between the photometric scale factor and systematic photometric errors (Research Note)
}

\author{
D. M. Bramich, E. Bachelet, K. A. Alsubai, D. Mislis, and N. Parley
}

Qatar Environment and Energy Research Institute, Qatar Foundation, PO Box 5825, Doha, Qatar

e-mail: dan.bramich@hotmail.co.uk

Received 5 March 2015 / Accepted 9 April 2015

\section{ABSTRACT}

\begin{abstract}
Context. Understanding the source of systematic errors in photometry is essential for their calibration.
Aims. We investigate how photometry performed on difference images can be influenced by errors in the photometric scale factor. Methods. We explore the equations for difference image analysis (DIA), and we derive an expression describing how errors in the difference flux, the photometric scale factor and the reference flux are propagated to the object photometry.

Results. We find that the error in the photometric scale factor is important, and while a few studies have shown that it can be at a significant level, it is currently neglected by the vast majority of photometric surveys employing DIA.

Conclusions. Minimising the error in the photometric scale factor, or compensating for it in a post-calibration model, is crucial for reducing the systematic errors in DIA photometry.
\end{abstract}

Key words. techniques: photometric - techniques: image processing - methods: data analysis

\section{Introduction}

The technique of difference image analysis (DIA; Alard \& Lupton 1998; Alard 2000; Bramich 2008; Becker et al. 2012; Bramich et al. 2013) is based on matching a reference image to a target image by modelling the differences in alignment, pointspread function (PSF), exposure time, atmospheric extinction, and sky background between them. Specifically, a convolution kernel is used to model the first four differences (to within a small translational offset) while an additive differential background is used to model the last. The reference image is transformed by convolving it with the kernel and adding the differential background, and the result is subtracted from the target image to create a difference image. All non-varying sources are fully subtracted in the difference image, leaving a signal only for sources that have varied in brightness (or possibly position).

Difference image analysis is being increasingly used for precision photometry and transient detection in a wide range of photometric surveys (e.g. PanSTARRS - Kaiser et al. 2002; OGLE - Udalski 2003; LSST - Ivezić et al. 2008; RoboNet-II - Tsapras et al. 2009; QES - Alsubai et al. 2013). Furthermore, these surveys are investing substantial efforts into post-calibration to minimise the systematic noise in the survey photometry that affects important aspects such as the detection limits, homogeneity, and completeness (e.g. Stubbs et al. 2010; Ofek et al. 2012; Wittman et al. 2012). Therefore, it is crucial to understand how the difference images, on which the photometry is performed, are created and how systematic errors in the difference images themselves can affect the photometry. However, there is no study in the literature on the systematic errors specific to DIA. In this research note, we have opened the investigation into systematic errors in DIA by exploring the effect that an error in the kernel sum, known as the photometric scale factor, may have on the photometry.

\section{Equations}

The target image $I$ is modelled as the convolution of a reference image $R$ with a convolution kernel plus a differential background. If one considers the kernel as the product of a photometric scale factor $P$ and a normalised (by its sum) kernel $K$, then the model target-image $M$ is defined as follows:

$M=P(R \otimes K)+B$.

The corresponding difference image $D$ is the image of model residuals given by

$$
\begin{aligned}
D & =I-M \\
& =I-P(R \otimes K)-B .
\end{aligned}
$$

Now assume that an object consisting of a source of interest and a blend has a true flux $f(t)$ on the photometric scale of the reference image that is given by

$f(t)=\left(1+\frac{k(t)}{1+k_{\mathrm{b}}}\right)\left(1+k_{\mathrm{b}}\right) f_{\mathrm{S}}$,

where $k(t)$ is a function of time $t$ that represents any variability in the source and $k_{\mathrm{b}}$ is the true blend ratio. Without loss of generality, adopt $k(0)=0$ so that the quantity $f_{\mathrm{S}}$ represents the true source flux at $t=0$. The true blend flux $k_{\mathrm{b}} f_{\mathrm{S}}$ makes the source appear brighter by a factor of $\left(1+k_{\mathrm{b}}\right)$ and reduces the apparent fractional flux-amplitude of any source variability by the same factor. 
It follows that the true object flux $f_{R}$ on the reference image taken at $t=0$ is

$f_{R}=\left(1+k_{\mathrm{b}}\right) f_{\mathrm{S}}$,

and that the true object flux $f_{\mathrm{I}}(t)$ on the target image taken at time $t$ is

$f_{\mathrm{I}}(t)=P f(t)$.

By considering Eq. (2), the true object flux $f_{D}(t)$ on the difference image may be computed as

$f_{D}(t)=f_{\mathrm{I}}(t)-P f_{R}$

By substituting Eq. (5) into Eq. (6) and rearranging, $f(t)$ may be written in terms of the measurable quantities $f_{R}$ and $f_{D}(t)$ as

$f(t)=f_{R}+f_{D}(t) / P$.

This is the equation used to convert difference fluxes into total fluxes and thence magnitudes (e.g. Bramich et al. 2011).

Unfortunately, the modelling of the target image is never perfect, and the difference image consequently suffers from small systematic errors that are propagated to the photometry. An error in the fitted differential background that results in a nonzero background in the difference image is trivially accounted for, at the expense of a little extra variance in the photometry, by including the background as a parameter in the method used to perform the photometry on the difference image (i.e. PSF fitting or aperture photometry). However, an error in the PSF matching (both shape and scale) produces systematic residuals at the object positions that are more difficult to mitigate at the image-processing stage. Aperture photometry is agnostic to mismatches in PSF shape, but will be affected by an error in the fitted photometric scale factor. PSF photometry, on the other hand, is sensitive to mismatches in both PSF shape and scale, but will provide photometry with smaller variance than aperture photometry when stochastic noise dominates. For these reasons, we ignore the error in the fitted differential background, we treat the error in the fitted normalised kernel as part of the error introduced into the photometry by the measurement process on the difference image, and we assume that the fitted photometric scale factor suffers from a small fractional error.

We use $P^{\prime}, K^{\prime}$, and $B^{\prime}$ to represent the fitted photometric scale factor, kernel, and differential background. Employed in Eq. (2), these give us the difference image $D^{\prime}$ with systematic errors. If we assume that the difference flux is measured with an error of $\varepsilon_{D} f_{\mathrm{I}}(t)$ due to stochastic noise and/or the error in $K^{\prime}$, and if we also assume that the error in $B^{\prime}$ can be successfully accounted for, then we obtain the following measured difference flux on $D^{\prime}$ for the object:

$f_{D^{\prime}}^{\prime}(t)=f_{\mathrm{I}}(t)-P^{\prime} f_{R}+\varepsilon_{D} f_{\mathrm{I}}(t)$.

Adopting the expression $P^{\prime}=\left(1+\varepsilon_{P}\right) P$ for the relation between $P^{\prime}$ and $P$, and using Eqs. (3) to (5), we may derive

$f_{D^{\prime}}^{\prime}(t)=P\left(\left(1+\varepsilon_{D}\right) k(t)+\left(\varepsilon_{D}-\varepsilon_{P}\right)\left(1+k_{\mathrm{b}}\right)\right) f_{\mathrm{S}}$.

If we now assume that the method of performing photometry on the reference image yields a fractional flux error of $\varepsilon_{R}$ in the reference flux that is different from $\varepsilon_{D}$ because of the different nature of the reference image and/or method used, then the measured reference flux is $f_{R}^{\prime}=\left(1+\varepsilon_{R}\right) f_{R}$. Using $f_{R}^{\prime}, f_{D^{\prime}}^{\prime}(t)$ and $P^{\prime}$ in Eq. (7) and doing some algebra yields the following expression for the measured object flux $f^{\prime}(t)$ on the photometric scale of the reference image:

$f^{\prime}(t)=\left(1+\frac{k(t)}{(1+\delta)\left(1+k_{\mathrm{b}}\right)}\right)\left(\frac{1+\varepsilon_{D}}{1+\varepsilon_{P}}\right)(1+\delta)\left(1+k_{\mathrm{b}}\right) f_{\mathrm{S}}$,

where

$\delta=\varepsilon_{R}\left(\frac{1+\varepsilon_{P}}{1+\varepsilon_{D}}\right)$

Equation (10) describes how the measured flux of a constant source $(k(t)=0$ for all $t)$, or a variable source $(k(t) \neq 0$ for at least some $t)$, is distorted by the errors $\varepsilon_{D}, \varepsilon_{P}$, and $\varepsilon_{R}$. The equation has been written in the form above to facilitate direct comparison to Eq. (3), which represents the true object flux. The ratio of the measured to the true object flux is

$\frac{f^{\prime}(t)}{f(t)}=\left(1+\frac{\delta}{1+\frac{k(t)}{1+k_{\mathrm{b}}}}\right)\left(\frac{1+\varepsilon_{D}}{1+\varepsilon_{P}}\right)$.

In magnitudes, Eqs. (3) and (10) become

$$
\begin{aligned}
m(t)=- & 2.5 \log \left(f_{\mathrm{S}}\right)-2.5 \log \left(1+\frac{k(t)}{1+k_{\mathrm{b}}}\right)-2.5 \log \left(1+k_{\mathrm{b}}\right) \\
m^{\prime}(t)= & -2.5 \log \left(f_{\mathrm{S}}\right)-2.5 \log \left(1+\frac{k(t)}{(1+\delta)\left(1+k_{\mathrm{b}}\right)}\right) \\
& -2.5 \log \left(1+\varepsilon_{D}\right)+2.5 \log \left(1+\varepsilon_{P}\right) \\
& -2.5 \log (1+\delta)-2.5 \log \left(1+k_{\mathrm{b}}\right)
\end{aligned}
$$

where $m(t)$ and $m^{\prime}(t)$ are the true and measured object magnitudes, respectively, on the magnitude scale of the reference image.

\section{Discussion}

The difference flux $f_{D}(t)$ is a quantity that is measured for each object on each difference image, and therefore $\varepsilon_{D}$ is specific to the object and difference image under consideration. However, for multiple difference images, any systematic (as opposed to stochastic) component in $\varepsilon_{D}$ that is a function of either an object property (e.g. colour) and/or an image property (e.g. pixel coordinates) may be estimated by solving for the appropriate magnitude offsets using the DIA photometry of all of the constant objects in the corresponding target images. This approach was developed by authors such as Honeycutt (1992) and Manfroid (1995) and is starting to be adopted by many surveys as the standard procedure for performing a post-calibration of the photometric data (e.g. Padmanabhan et al. 2008). In this respect, post-calibration of DIA photometry is no different than the postcalibration of photometry performed directly on the target images. The appropriate magnitude offsets to be determined from the constant objects are represented by the term $-2.5 \log \left(1+\varepsilon_{D}\right)$ in Eq. (14) and their absolute values are usually of the order of 1-30 mmag.

The reference flux $f_{R}$ is a quantity that is measured for each object on the reference image. Therefore the error $\varepsilon_{R}$ is independent of the target image (or time) and it affects the photometry of constant objects by making them systematically too bright $\left(\varepsilon_{R}>0\right)$ or too faint $\left(\varepsilon_{R}<0\right)$. Variable objects suffer this same systematic error, and in addition, their fractional flux-amplitude of variation is either systematically amplified $\left(\varepsilon_{R}<0\right)$ or reduced $\left(\varepsilon_{R}>0\right)$ by a factor of $(1+\delta) \sim\left(1+\varepsilon_{R}\right)$ to first order. In 
this respect, the effect of $\varepsilon_{R}$ is equivalent to that of an extra blend flux. The absolute value of $\varepsilon_{R}$ is usually in the range of typical photometric precisions of $\sim 0.1-5 \%$. Unless the DIA photometry has a very low stochastic noise component $(\$ 0.01 \%), \varepsilon_{R}$ is indistinguishable from $k_{\mathrm{b}}$ since its effect on $f^{\prime}(t)$ only differs from that of $k_{\mathrm{b}}$ to second order (see Eqs. (10) and (11)). We do not consider any further the intricacies of disentangling the source flux, blend ratio, and reference flux error. We note, however, that when the blend ratio can be determined (e.g. by using external information, or for certain types of variability such as microlensing events), the estimated blend ratio $k_{\mathrm{b}}^{\prime}$ is related to the true blend ratio by $k_{\mathrm{b}}^{\prime} \sim\left(1+\varepsilon_{R}\right) k_{\mathrm{b}}+\varepsilon_{R}$.

When fitting the model target-image, the photometric scale factor $P$ is typically assumed to be spatially invariant and hence is characterised by a single number, although $P$ may also be modelled as a function of detector coordinates (Bramich et al. 2013). Regardless of how $P$ is modelled, the error $\varepsilon_{P}$ will be different for each target image since it is determined on a per-image basis from noisy images. Other effects such as flatfielding errors, or changing non-uniform atmospheric extinction (i.e. clouds and/or airmass gradients), may conspire to make $\varepsilon_{P}$ specific to the object and target image under consideration. As with $\varepsilon_{D}$, some of the systematic components in $\varepsilon_{P}$ may be estimated by solving for the appropriate magnitude offsets using the DIA photometry of all of the constant objects in the target images. In fact, the systematic components in $\varepsilon_{P}$ that are also common to those in $\varepsilon_{D}$ may be absorbed into the magnitude offsets represented by the term $-2.5 \log \left(1+\varepsilon_{D}\right)$ in Eq. (14). However, the remaining systematic components in $\varepsilon_{P}$, if left uncorrected, will cause errors in the DIA photometry that have the potential of being misinterpreted as real signals.

\section{Summary and recommendations}

The vast majority of photometric surveys employing DIA do not consider $P$ to be a source of error since it is assumed that $P$ has been precisely determined, which is equivalent to assuming that the differences in atmospheric extinction between the reference and target images have been perfectly accounted for by the DIA modelling itself. However, in this research note we have shown that any errors in $P$ that do exist will have an important effect on the DIA photometry. We therefore strongly recommend that it becomes standard procedure to assess and, if necessary, correct for the effect of the mean error in $P$ for each target image on the DIA photometry (encapsulated by the term $2.5 \log \left(1+\varepsilon_{P}(t)\right)$ in Eq. (14)).

One method to do this involves fitting a post-calibration photometric model including a set of per-image magnitude offsets $2.5 \log \left(1+\varepsilon_{P}(t)\right)$ to the DIA photometry of all of the constant objects in the target images. Note that the post-calibration model should also include the magnitudes of the constant objects as free parameters (see Bramich \& Freudling 2012) and any other relevant terms such as $-2.5 \log \left(1+\varepsilon_{D}\right)$. If the variations in the per-image magnitude offsets are found to be smaller than the level of the stochastic noise in the best object photometry, then they may be dropped from the post-calibration model. However, if they are deemed to be significant, then the per-image magnitude offsets $2.5 \log \left(1+\varepsilon_{P}(t)\right)$ may be used to correct the DIA photometry of all of the objects for the photometric error introduced by $\varepsilon_{P}(t)$.

This technique has started to be used in the series of papers on variable stars in globular clusters by the lead author (e.g. Kains et al. 2013; Arellano Ferro et al. 2013, which employ the methodology of Bramich \& Freudling 2012), and in these works the relevant per-image magnitude offsets are found to be at the $0.1-2 \%$ level (see Fig. 1 of Kains et al. 2015). This example clearly demonstrates that DIA does not always perform the photometric matching between images to a precision that is below the stochastic noise in the photometric measurements, and it serves to emphasise how crucial it is to account for the mean error in $P$ for each target image to minimise the associated systematic errors.

Finally we caution that when low-level $(\$ 1-2 \%)$ suspected signals occur in the DIA photometry of an object that cannot be confirmed either by the detection of a repeating signal (for periodic signals) or by independent observations taken at the same epoch, a careful analysis of the images on which the suspected signal was detected is warranted. It needs to be determined whether there were any clouds (e.g. light cirrus) that may have caused non-uniform atmospheric extinction across the field of view that also evolves throughout the time series with the cloud movement since this can cause smooth temporal variations in $\varepsilon_{P}$ that are different for each object and that will manifest themselves as smooth variations in the object light curves. Adopting a spatially variable photometric scale factor in the model targetimage for the DIA may partly mitigate this problem.

Acknowledgements. This publication was made possible by NPRP grant \# X019-1-006 from the Qatar National Research Fund (a member of Qatar Foundation). The statements made herein are solely the responsibility of the authors.

\section{References}

Alard, C. 2000, A\&AS, 144, 363

Alard, C., \& Lupton, R. H. 1998, ApJ, 503, 325

Alsubai, K. A., Parley, N. R., Bramich, D. M., et al. 2013, Acta Astron., 63, 465 Arellano Ferro, A., Bramich, D. M., Figuera Jaimes, R., et al. 2013, MNRAS, 434, 1220

Becker, A. C., Homrighausen, D., Connolly, A. J., et al. 2012, MNRAS, 425, 1341

Bramich, D. M. 2008, MNRAS, 386, L77

Bramich, D. M., \& Freudling, W. 2012, MNRAS, 424, 1584

Bramich, D. M., Figuera Jaimes, R., Giridhar, S., \& Arellano Ferro, A. 2011, MNRAS, 413, 1275

Bramich, D. M., Horne, K., Albrow, M. D., et al. 2013, MNRAS, 428, 2275

Honeycutt, R. K. 1992, PASP, 104, 435

Ivezić, Ž., Axelrod, T., Brandt, W. N., et al. 2008, Serb. Astron. J., 176, 1

Kains, N., Bramich, D. M., Arellano Ferro, A., et al. 2013, A\&A, 555, A36

Kains, N., Arellano Ferro, A., Figuera Jaimes, R., et al. 2015, A\&A, in press, DOI: $10.1051 / 0004-6361 / 201424600$

Kaiser, N., Aussel, H., Burke, B. E., et al. 2002, Proc. SPIE, 4836, 154

Manfroid, J. 1995, A\&AS, 113, 587

Ofek, E. O., Laher, R., Law, N., et al. 2012, PASP, 124, 62

Padmanabhan, N., Schlegel, D. J., Finkbeiner, D. P., et al. 2008, ApJ, 674, 1217

Stubbs C. W., Doherty, P., Cramer, C., et al. 2010, ApJS, 191, 376

Tsapras, Y., Street, R., Horne, K., et al. 2009, Astron. Nachr., 330, 4

Udalski, A. 2003, Acta Astron., 53, 291

Wittman, D., Ryan R., \& Thorman P., 2012, MNRAS, 421, 2251 